\title{
Suppression of GSK3 $\beta$ by ERK mediates lipopolysaccharide induced cell migration in macrophage through $\beta$-catenin signaling
}

\author{
Kai Gong ${ }^{1^{*}}$, Fangfang Zhou ${ }^{1,2^{*} \bowtie}$, Huizhe Huang ${ }^{1,3}$, Yandao Gong ${ }^{1}$, Long Zhang ${ }^{2}$ \\ ${ }_{1}^{1}$ Department of Biological Sciences and Biotechnology, Tsinghua University, Beijing 100084, China \\ ${ }^{2}$ Leiden University Medical Center, Department of Molecular Cell Biology, Leiden, the Netherland \\ ${ }^{3}$ Faculty of Basic Medical Sciences, Chongqing Medical University, Chongqing 400016, China \\ $\triangle$ Correspondence: L.zhang@lumc.nl (L. Zhang), F.zhou@lumc.nl (F. Zhou) \\ Received June 10, 2012 Accepted August 27, 2012
}

\begin{abstract}
We investigate the role of $\beta$-catenin signaling in the response of macrophage to lipopolysaccharide (LPS) using RAW264.7 cells. LPS rapidly stimulated cytosolic $\beta$-catenin accumulation. $\beta$-catenin-mediated transcription was showed to be required for LPS induced gene expression and cell migration. Mechanically, ERK activation-primed GSK3 $\beta$ inactivation by Akt was demonstrated to mediate the LPS induced $\beta$-catenin accumulation. Overall, our findings suggest that suppression of GSK3 $\beta$ by ERK stimulates $\beta$-catenin signaling therefore contributes to LPS induced cell migration in macrophage activation.
\end{abstract}

KEYWORDS LPS, ERK, GSK3 $\beta, \beta$-catenin, cell migration

\section{INTRODUCTION}

Bacterial lipopolysaccharide (LPS) triggers a series of signaling events in innate immune responses. LPS binds to LBP and membrane-bounded CD14 and associates with Toll-like receptor 4 (TLR4) (Poltorak et al., 1998; Means et al., 1999; Lien et al., 2000). CD14 mediated LPS induced activation of MEK/ERK signaling (Guha and Mackman, 2001). As the central recognition protein in the LPS receptor complex, TLR4 causes recruitment of MyD88, IRAK, and TRAF6 to the receptor where TRAF6 is catalyzed synthesis of K63-linked polyubiquitin chains and subsequently activates NF-KB (Lomaga et al., 1999; Carpenter and O'Neill, 2009).

In contact with bacterial infection, immune cells such as macrophage play essential role to initiate defensive re-

\footnotetext{
*These authors contributed equally to the work.
}

sponses, including releasing a large number of inflammation mediators and activating a number of signaling cascades. Wnt pathway is one of the signaling cascades in macrophages. As a family of secreted proteins, Wnt regulate cell proliferation, migration and differentiation (Logan and Nusse, 2004; Moon et al., 2004; Reya and Clevers, 2005; MacDonald et al., 2009; Zhang and $\mathrm{Ma}, 2010)$. One of the major components of the canonical Wnt-induced signaling is $\beta$-catenin, which is rapidly degraded by the proteasome system due to phosphorylation by the Adenomatous polyposis coli (APC)-Axin-glycogen synthase kinase (GSK) $3 \beta$ complex (Liu et al., 2002; Nelson and Nusse, 2004). Blocking GSK3 $\beta$ kinase activity, such as phosphorylation by AKT (Cross et al., 1995), leads to the cytosolic accumulation of $\beta$-catenin that freely enters into the nucleus and triggers target gene activation by displacing transcriptional repressors from DNA-bound LEF/TCF (Behrens et al., 1996; Brunner et al., 1997). The present work describes the role of ERK-mediated activation of canonical Wnt/ 3 -catenin signaling and its role in LPS induced macrophage activation.

\section{RESULTS}

LPS stimulation induces $\beta$-catenin accumulation and promotes cell migration in macrophage

We investigated Wnt/ $\beta$-catenin signaling in LPS-treated cells by western blotting and q-PCR analysis. Exposure of RAW264.7 cells to LPS markedly increased total and cytosolic $\beta$-catenin in a time course dependent manner (Fig. 1A). The $\beta$-catenin induction pattern is negative correlated with GSK3 $\beta$ kinase activity and positively correlated with AKT and 


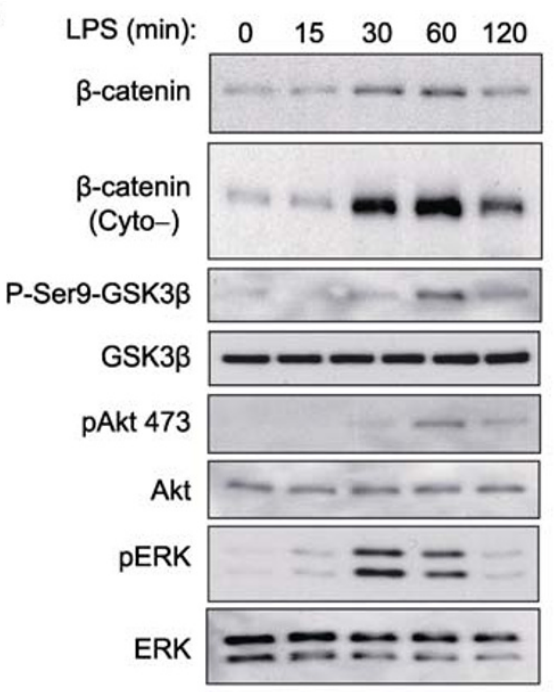

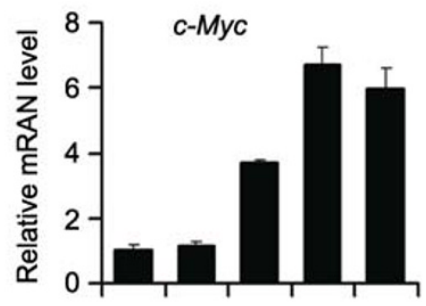

LPS (min): $0 \quad 30 \quad 60 \quad 120 \quad 240$

C

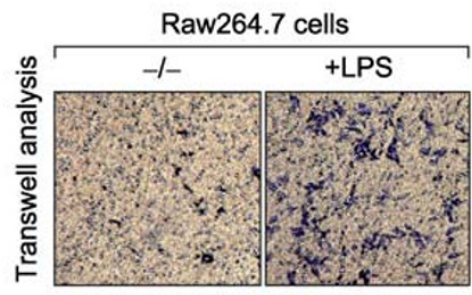

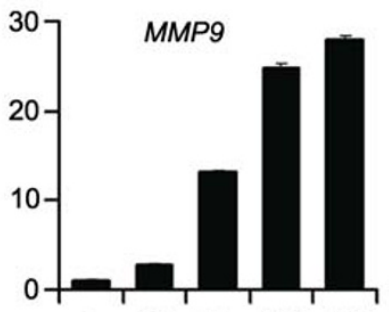

$0 \quad 30 \quad 60 \quad 120 \quad 240(\mathrm{~min})$

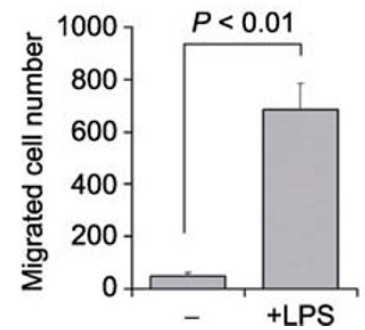

Figure 1. LPS stimulation induces $\beta$-catenin accumulation and promotes cell migration in macrophage. (A) Immunoblot analysis in RAW264.7 cells treated with LPS $(100 \mathrm{ng} / \mathrm{mL})$ as indicated. (B) qPCR analysis of $c-M y c$ and MMP9 expressions in LPS stimulated RAW264.7 cells as indicated. (C) LPS (100 ng/mL) promotes RAW264.7 cell migration using transwell migration essay. Migrated cell number was quantified in the right panel.

ERK activations by LPS (Fig. 1A). Q-PCR analysis showed that LPS induced expression of $\beta$-catenin direct target genes $c-M y c$ and MMP9 (Fig. 1B). Consistent with the MMP9 induction, functionally as measured in transwell migration assay, LPS sharply potentiated macrophage cell motility (Fig. 1C), the characteristic that enables infiltration from blood vessels and cell migration for antigens, contributing strategically to the defending inflammation process.

\section{Blocking $\beta$-catenin mediated transcription impairs LPS induced MMP9 expression and reduces cell migration}

To test the role of $\beta$-catenin-mediated transcription in LPS induced gene expression and macrophage migration. Dominant negative LEF-1(dnLEF-1) was introduced into RAW cells to specifically block $\beta$-catenin mediated transcription. As shown in Fig. 2A, dnLEF-1 inhibited both the basal and LPS induced c-Myc and MMP9 gene expressions. Consistently in the measurement of cell motility, dnLEF-1 significantly reduced LPS induced macrophage migration (Fig. 2B). Similar effects were also observed by using specific siRNA against $\beta$-catenin (Fig. 2). These results indicate the requirement of $\beta$-catenin mediated transcription for LPS induced macrophage activation. Consistently, treatment of GSK $\beta$ kinase inhibitor SB216763 or $\mathrm{LiCl}$ increased $\beta$-catenin accumulation and elevated macrophage cell migration (Fig. 2C and data not shown).

\section{Activation of both ERK and AKT are required in LPS-induced $\beta$-catenin accumulation.}

Immunoblotting of Phospho-ERK (p-ERK) and phosphor-AKT
473 ( $p-A K T$ 473) has been shown to be correlated with LPS triggered $\beta$-catenin accumulation, suggesting that activation of ERK and AKT pathways may be involved in this process. To test this hypothesis, ERK and AKT kinase inhibitors were employed to uncover the mechanism underlying. As shown in Fig. 3A, treatment of RAW cells with either ERK inhibitor or Akt inhibitor, (PD95059 and Ly294002 respectively) can partially block $\beta$-catenin accumulation by LPS. Combined treatment of both inhibitors almost completely blocked LPS stimulated $\beta$-catenin accumulation, an observation similar with infection of constitutive active GSK3 $\beta$ mutant (S9A) (Fig. $3 B$ ). Thus both ERK and AKT pathways are involved in $\beta$-catenin accumulation by LPS.

\section{Activated ERK associates with and primes GSK3 $\beta$ inactivation by AKT in response to LPS}

Combined together, AKT activation (as viewed by pAkt 473) mediated GSK3 $\beta$ inactivation (as viewed by pSer9 GSK3 $\beta$ ) is likely to be responsible for LPS induced $\beta$-catenin accumulation. Thus we investigate the role of ERK. Since ectopic tagged ERK and GSK3 $\beta$ could interact with each other in HEK293T cells (data not shown), we hypothesized that endogenous ERK may regulate GSK3 $\beta$ activity via association. We first performed co-immunoprecipitation assays to test whether ERK can interact with GSK3 $\beta$ in macrophage cells. The endogenous ERK in RAW cells could indeed be co-precipitated with endogenous GSK3 $\beta$ (Fig. 4A). Notably, LPS induced p-ERK associated with GSK3 $\beta$ and increased the level of GSK3 $\beta$ phosphorylation in Ser9 (Fig. 4A). Reversely, endogenous GSK3 $\beta$ was also found in p-ERK im- 
munoprecipitation in 30 minutes treatment point of LPS (Fig. 4B). These results suggested that LPS induced activation of ERK which may subsequently facilitate GSK3 $\beta$ inactivation by AKT. To consolidate this, we examined in ERK2 overxpressed RAW cells the GSK3 $\beta$ activity and its downstream $\beta$-catenin expression. As shown in Fig. $4 \mathrm{C}$, GSK3 $\beta$ is inactivated and $\beta$-catenin is indeed accumulated in cytosolic fraction of ERK overexpressed macrophage cells. Taken together, ERK associates with GSK3 $\beta$ and primes GSK3 $\beta$ inactivation in LPS treated macrophages.
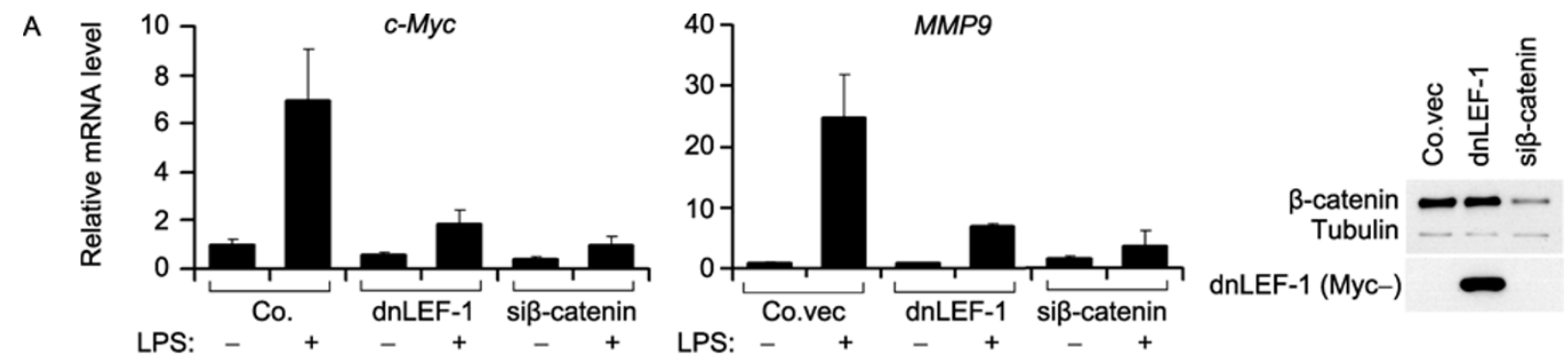

B

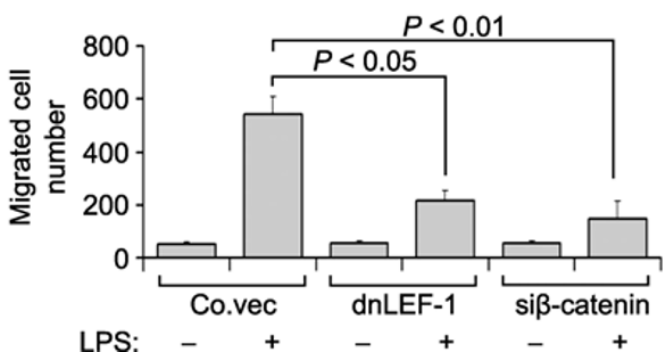

C

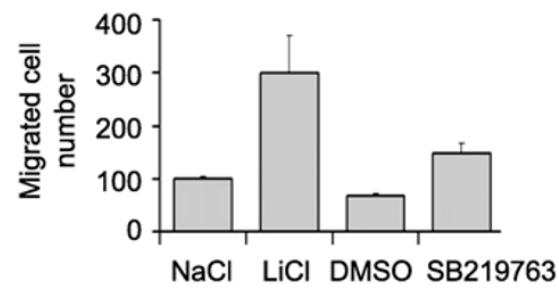

Figure 2. Ectopic expression of dominant negative LEF-1 impairs LPS induced c-Myc and MMP9 expression and reduces cell migration. ( $A$ and $B$ ) Control (Co.vector and Co.siRNA) and dnLEF-1 or siß-catenin transfected RAW264.7 cells were treated with or without LPS $(100 \mathrm{ng} / \mathrm{mL})$ for $4 \mathrm{~h}$ as indicated, cells were harvested for qPCR analysis $(A)$ or employed for transwell migration essay (B). Expression of Myc tagged dominant negative LEF-1 (dnLEF-1) and $\beta$-catenin knocking down effects was shown in Fig. 2A, right panel. (C) Transwell analysis of RAW264.7 cell migration in the presence or absence of GSK3 $\beta$ kinase inhibitor SB216763 $(10 \mu \mathrm{mol} / \mathrm{L})$ or $\mathrm{LiCl}(10 \mathrm{mmol} / \mathrm{L})$. Migrated cell number was quantified from 5 random fields.

A
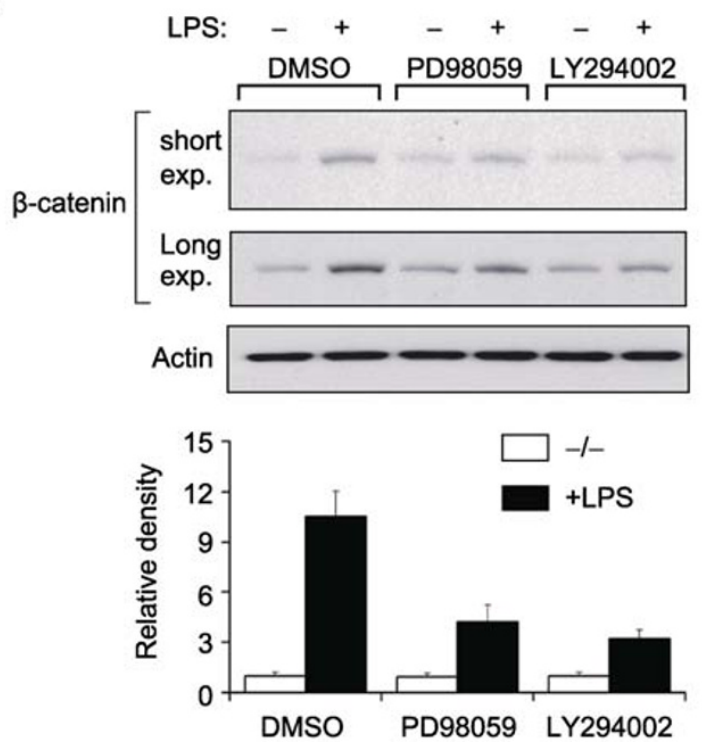
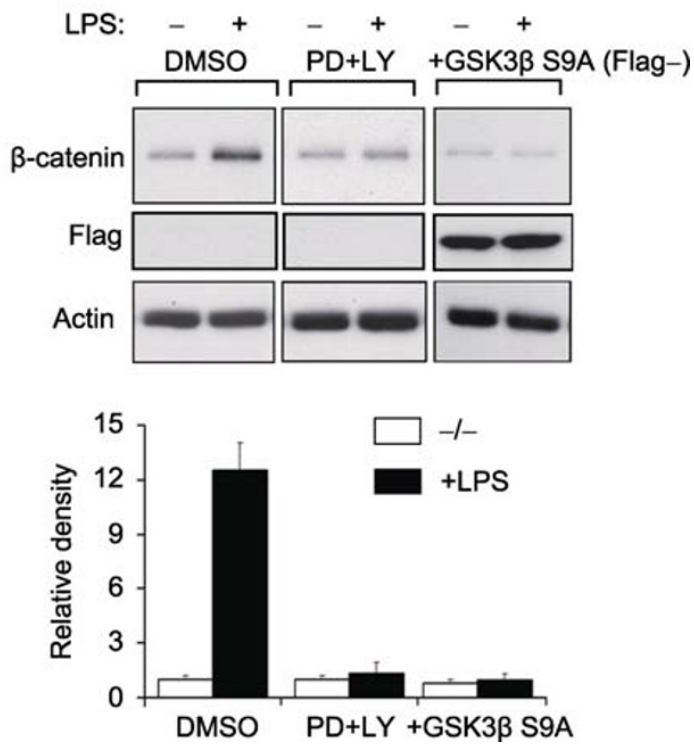

Figure 3. Activation of Erk and Akt are involved in LPS-induced $\beta$-catenin accumulation. (A) Control DMSO, PD98059 (10 $\mu \mathrm{mol} / \mathrm{L})$ or LY294002 $(10 \mu \mathrm{mol} / \mathrm{L})$ pretreated RAW264.7 cells were stimulated with or without LPS for 1 hour. Cells were harvest for immunoblottings. Statistically analysis was shown below $(n=3)$. (B) RAW264.7 cells were treated with control DMSO, combination of PD98059 $(10 \mu \mathrm{mol} / \mathrm{L})$ and LY294002 $(10 \mu \mathrm{mol} / \mathrm{L})$, or infected with Flag-GSK3 $\beta$ S9A lenti-virus. Cells were stimulated with or without LPS for $1 \mathrm{~h}$ and harvested for immunoblot analysis. Statistically analysis was shown below $(n=3)$. 
A

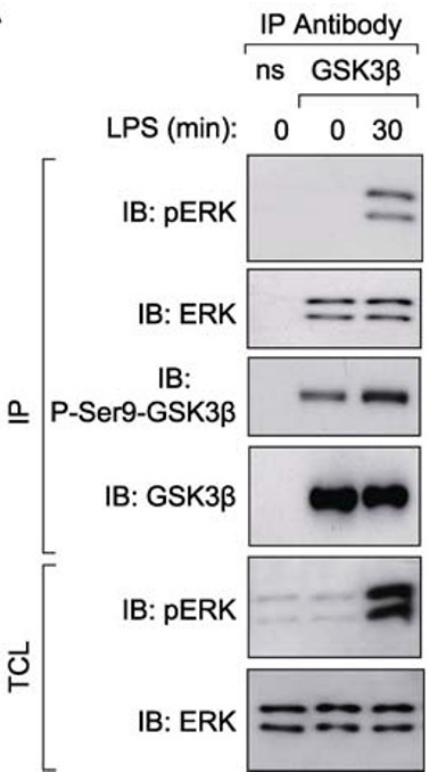

B

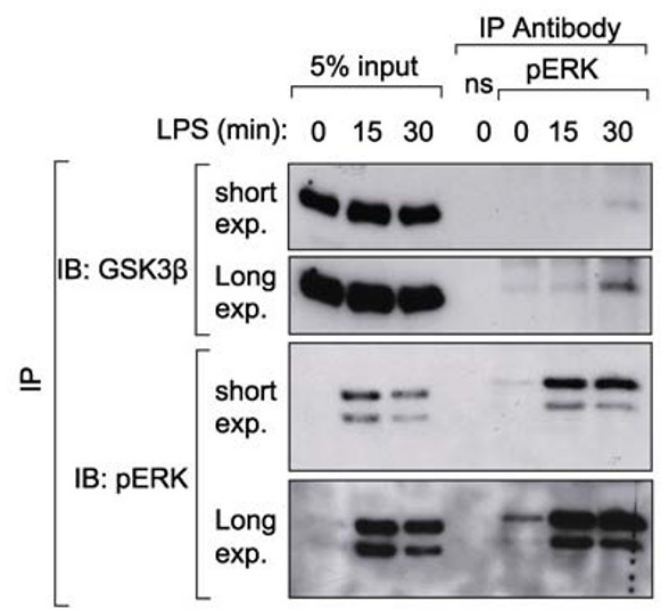

C

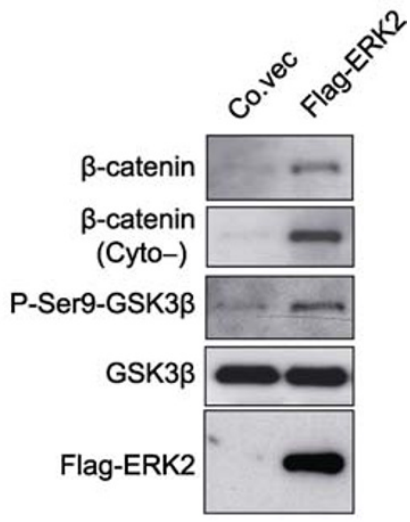

Figure 4. Erk associates with and primes GSK3 $\beta$ inactivation by Akt. (A) In the presence or absence of LPS (100 ng/mL) treatment, RAW264.7 cells were harvested for anti-GSK3 $\beta$ or control anti-none specific (ns) immunoprecipitation (IP) and immunoblottings (IB) as indicated. (B) Control and LPS stimulated RAW264.7 cells were harvested for anti-phosphor-ERK immunoprecipitation and immunoblottings as indicated. (C) Immunoblot analysis of Flag-ERK2 overexpressed RAW264.7 cells compared with control.

\section{ERK activated $\beta$-catenin pathway is required for LPS induced MMP9 expression and cell migration in macrophage}

To prove the possible role of ERK- $\beta$-catenin cascades in LPS induced macrophage cell characteristics such as downstream gene activation and cell migratory capacity. We treated RAW cells with ERK inhibitor PD98059 and examined LPS induced
MMP9 expression and cell migration. Q-PCR analysis showed that PD98059 treatment significantly suppressed LPS induced MMP9 expression and blocked RAW cell migration (Fig. 5A and $5 B$ ). These effects were clearly rescued by ectopic expression of constitutive active $\beta$-catenin mutant (SY) (Fig 5A-C). Taken together, these results showed that ERK activated $\beta$-catenin pathway is required for LPS induced MMP9 expression and cell migration in macrophage.

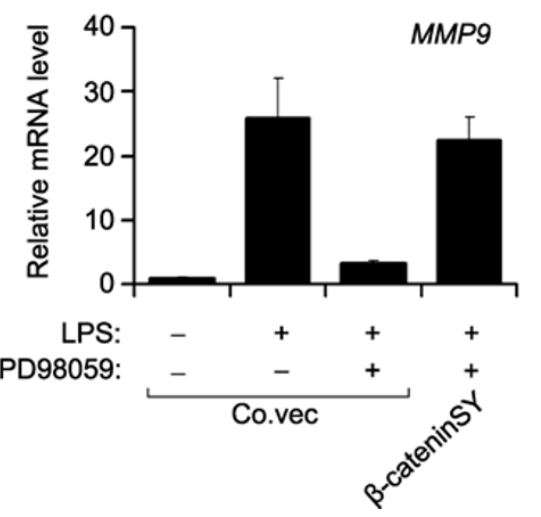

B

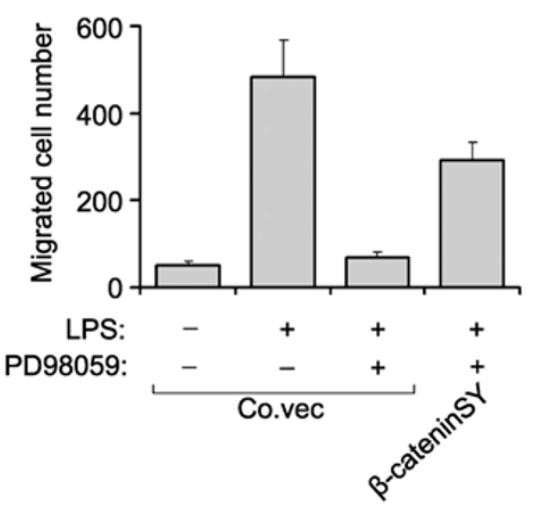

C

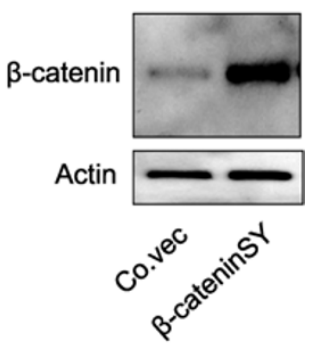

Figure 5. LPS induced MMP9 expression and cell migration is inhibited by ERK kinase inhibitor and rescued by ectopic $\beta$-catenin SY expression. (A and B) Control vector (Co.vec) and $\beta$-catenin SY infected RAW264.7 cells were treated with LPS or PD98059 $(10 \mu \mathrm{mol} / \mathrm{L})$ as indicated, cells were harvested for qPCR analysis (A) or cell migration essay (B). (C) $\beta$-catenin expression was examined by immunoblotting. 


\section{DISCUSSION}

As a signaling pathway with function in many aspects including cell proliferation, migration and differentiation, the Wnt/ß-catenin pathway is tightly controlled by regulators at multiple steps, the amount of (cytosolic) $\beta$-catenin being the most decisive response parameter. In this study, we demonstrated that LPS rapidly induced $\beta$-catenin accumulation in macrophage. We confirmed the activation of this pathway by examining its downstream direct target genes, such as $c-M y c$ and MMP9. As one of the MMPs, MMP9 is released by macrophage to degrade Collagen, Elastin, Fibronectin and other extracellular matrix components, which is important for migration. To elucidate the requirement of $\beta$-catenin in LPS induced effect, we specifically blocked $\beta$-catenin mediated transcription by expressing dnLEF-1 or siß-catenin in macrophages. This indeed impaired LPS induced MMP9 expressin and macrophage motility.

We next examined the mechanism underlying. Dickkopf-1 conditioned medium failed to block LPS induced $\beta$-catenin accumulation and LRP6 phosphorylation (Ser1490) was not observed in the presence of LPS in macrophage cells (data not shown), suggesting Wnt receptor activation is not responsible for LPS induced $\beta$-catenin activation. However, correlated to LPS triggered $\beta$-catenin accumulation, we observed inactivation of GSK3 $\beta$ and activation of both AKT and ERK. Moreover, both AKT and ERK inhibitors were showed to block $\beta$-catenin accumulation by LPS. Endogenous ERK was found to associate with GSK3 $\beta$ and facilitate its inactivation by AKT, as detected in pSer9 GSK3 $\beta$ level. Additionally, degradation resistant $\beta$-catenin active form rescued ERK inhibitor mitigated MMP9 gene induction and cell migration by LPS. This further confirmed the finding that ERK potentiated $\beta$-catenin signaling cascades in LPS induced macrophage cells. Our finding supports a previous observation in hepatocellular carcinoma that ERK is activated to activate $\beta$-catenin signaling (Ding et al., 2005).

Release of MMP molecules and initiation of macrophage migration are important for host defense and direction of the adaptive immune system. When uncontrolled, they can cause significant damage on the microenvironment. By eliciting massive inflammatory mediators, pro-apoptotic factors, and matrix degrading proteases, the activated macrophage is capable of dismantling tissues to the point of inflicting serious injury. Tissue destruction by chronic inflammation has been associated with the development of tumors and other pathologies (Gordon, 2003). Therefore, the turning off of LPS induces $\beta$-catenin signaling is even more interesting than the "turning on", which is left to be studied in the future.

In conclusion, the results of the present study suggested that, in LPS stimulated macrophages, the $\beta$-catenin and its downstream signals play strategic roles in inducing cell mobility. $\beta$-catenin signaling contributes the early phage MMP9 gene induction and cell migration. Mechanistically, rapid ERK activation primed GSK3 $\beta$ inactivation by AKT is involved in efficient silencing $\beta$-catenin destruction complex by LPS.

\section{MATERIALS AND METHODS}

\author{
Reagent and plasmids
}

$\beta$-catenin antibody (Cat. \#610153) was purchased from BD Biosciences; ERK(\#4695), pERK(\#9106), GSK3ß(\#9832), pSer9 GSK3ß(\#9323), Akt(\#4685), pAkt 473(\#4058) and Actin(\#4970) were purchased from Cell Signaling Technology, Inc (USA); LPS, LY294002, PD98059 were purchased from Sigma, USA. $\beta$-catenin SY, dnLEF-1 and GSK3 $\beta$ S9A and control constructs were previously described (Zhang et al., 2006, 2012a, 2012b, 2012c; Zhou et al., 2012a, 2012b).

\section{Cell culture}

RAW264.7 cells were cultured in RPMI 1640 medium supplemented with $10 \%$ fetal bovine serum and incubated at $37^{\circ} \mathrm{C}$ in a humidified atmosphere of $5 \% \mathrm{CO}_{2}$.

\section{Transwell migration assay}

As previously described (Zhang et al.,2012d,2012e), transwell assay were performed in 24-well PET inserts (Falcon $8.0 \mu \mathrm{m}$ pore size) for migration assays. 50,000 cells were plated in transwell inserts (at least 3 replicas for each sample) and left treated with or without LPS $(100 \mathrm{ng} / \mathrm{mL})$ for $4 \mathrm{~h}$. Cells in the upper part of the transwells were removed with a cotton swab; migrated cells were fixed in PFA $4 \%$ and stained with crystal Violet $0.5 \%$. Filters were photographed and the total number of cells counted. Every experiment was repeated at least three times independently.

\section{Real-time reverse transcription (RT)-PCR}

As previously described (Zhang et al., 2012b; Zhou et al., 2011a, 2011b), total RNAs were prepared using NucleoSpin® RNA II kit. Expression level of target genes was normalized with GAPDH. Primers used for qRT-PCR are listed as follows: mGAPDH forward, 5'-AACTTTGGCATTGTGGAAGG-3'; mGAPDH reverse, 5'-ACACATTGGGGGTAGGAACA-3'; mMMP9 forward, 5'-CAAGTGGGACCATCATAACATCA-3'; mMMP9 reverse, 5'-TCTCGCGGCAAGT CTTCAG-3'; mc-Myc forward, 5'-GTCTTTCCCTACCCGCTCAAC-3'; mc-Myc reverse, 5'-GTGGAATCGGACGAGGTACAG-3'

\section{Immunoprecipitation and immunoblotting}

Immunoprecipitation was performed as previously described (Zhang et al., 2004; Nie et al., 2010; Zhang et al., 2012b; Zhou et al., 2012b); Immunoblotting was performed with specific primary antibody as indicated and secondary anti-mouse or anti-rabbit antibodies that were conjugated to horseradish peroxidase (Amersham Biosciences). Proteins were visualized by chemiluminescence.

\section{Lentiviral transduction}

Lentiviruses were produced by transfecting pLV-bc-CMV (for cDNA 
expression) plasmids together with helper plasmids pCMV-VSVG, pMDLg-RRE (gag/pol), and pRSV-REV into HEK293T cells. Cell supernatants were harvested $48 \mathrm{~h}$ after transfection and were either used to infect cells or stored at $-80^{\circ} \mathrm{C}$

\section{Preparation of cytosolic and nuclear fractions}

Cytosolic fractions were prepared/isolated using the ProteoExtract kit (Calbiochem) according to the manufacturers' standard procedures.

\section{Statistical analysis}

Values are expressed as the mean \pm SE derived from 3 samples and the figures show the results obtained from three independent experiments. Statistical analysis was performed using analysis by Student's $t$-test and the level of significance was put at $P<0.01$ or $P<$ 0.05 as indicated.

\section{ACKNOWLEDGEMENTS}

We are grateful to Dr. Xiao-fan Wang of Duke University for GSK3 $\beta$ S9A construct. This work was supported by the Netherlands Organization for Scientific Research (NWO 918.66.066), Netherlands Initiative for Regenerative Medicine and Centre for Biomedical Genetics; CSTC2012 jjjq1000; and National Nature Science Foundation of China Grants 30900748 and 31171388 (to H. H.).

\section{ABBREVIATIONS}

APC, Adenomatous polyposis coli; dnLEF-1; Dominant negative LEF-1; GSK, glycogen synthase kinase; LPS, lipopolysaccharide; p-AKT, phosphor-AKT; p-ERK, Phospho-ERK; TLR4, Toll-like receptor 4

\section{REFERENCES}

Behrens, J., von Kries, J.P., Kuhl, M., Bruhn, L., Wedlich, D., Grosschedl, R., and Birchmeier, W. (1996). Functional interaction of beta-catenin with the transcription factor LEF-1. Nature 382, 638-642.

Brunner, E., Peter, O., Schweizer, L., and Basler, K. (1997). pangolin encodes a Lef-1 homologue that acts downstream of Armadillo to transduce the Wingless signal in Drosophila. Nature 385, 829-833.

Carpenter, S., and O'Neill, L.A. (2009). Recent insights into the structure of Toll-like receptors and post-translational modifications of their associated signalling proteins. Biochem J 422, 1-10.

Cross, D.A., Alessi, D.R., Cohen, P., Andjelkovich, M., and Hemmings, B.A. (1995). Inhibition of glycogen synthase kinase-3 by insulin mediated by protein kinase B. Nature 378, 785-789.

Ding, Q., Xia, W., Liu, J.C., Yang, J.Y., Lee, D.F., Xia, J., Bartholomeusz, G., Li, Y., Pan, Y., Li, Z., et al. (2005). Erk associates with and primes GSK-3beta for its inactivation resulting in upregulation of beta-catenin. Mol Cell 19, 159-170.

Gordon, S. (2003). Alternative activation of macrophages. Nat Rev Immunol 3, 23-35.
Guha, M., and Mackman, N. (2001). LPS induction of gene expression in human monocytes. Cell Signal 13, 85-94.

Lien, E., Means, T.K., Heine, H., Yoshimura, A., Kusumoto, S., Fukase, K., Fenton, M.J., Oikawa, M., Qureshi, N., Monks, B., et al. (2000). Toll-like receptor 4 imparts ligand-specific recognition of bacterial lipopolysaccharide. J Clin Invest 105, 497-504.

Liu, C., Li, Y., Semenov, M., Han, C., Baeg, G.H., Tan, Y., Zhang, Z., Lin, X., and He, X. (2002). Control of beta-catenin phosphorylation/degradation by a dual-kinase mechanism. Cell 108, 837-847.

Logan, C.Y., and Nusse, R. (2004). The Wnt signaling pathway in development and disease. Annu Rev Cell Dev Biol 20, 781-810.

Lomaga, M.A., Yeh, W.C., Sarosi, I., Duncan, G.S., Furlonger, C., Ho, A., Morony, S., Capparelli, C., Van, G., Kaufman, S., et al. (1999). TRAF6 deficiency results in osteopetrosis and defective interleukin-1, CD40, and LPS signaling. Genes Dev 13, 1015-1024.

MacDonald, B.T., Tamai, K., and He, X. (2009). Wnt/beta-catenin signaling: components, mechanisms, and diseases. Dev Cell 17, 9-26.

Means, T.K., Lien, E., Yoshimura, A., Wang, S., Golenbock, D.T., and Fenton, M.J. (1999). The CD14 ligands lipoarabinomannan and lipopolysaccharide differ in their requirement for Toll-like receptors. J Immunol 163, 6748-6755.

Moon, R.T., Kohn, A.D., De Ferrari, G.V., and Kaykas, A. (2004). WNT and beta-catenin signalling: diseases and therapies. Nat Rev Genet 5, 691-701.

Nelson, W.J., and Nusse, R. (2004). Convergence of Wnt, beta-catenin, and cadherin pathways. Science 303, 1483-1487.

Nie, J., Wang, H., He, F., and Huang, H. (2010). Nusap1 is essential for neural crest cell migration in zebrafish. Protein Cell 1, 259-266.

Poltorak, A., He, X., Smirnova, I., Liu, M.Y., Van Huffel, C., Du, X., Birdwell, D., Alejos, E., Silva, M., Galanos, C., et al. (1998). Defective LPS signaling in $\mathrm{C} 3 \mathrm{H} / \mathrm{HeJ}$ and $\mathrm{C} 57 \mathrm{BL} / 10 \mathrm{ScC}$ r mice: mutations in TIr4 gene. Science 282, 2085-2088.

Reya, T., and Clevers, H. (2005). Wnt signalling in stem cells and cancer. Nature 434, 843-850.

Zhang, B., and Ma, J.X. (2010). Wnt pathway antagonists and angiogenesis. Protein Cell 1, 898-906.

Zhang, J., Zhang, X., Zhang, L., Zhou, F., van Dinther, M., and Ten Dijke, P. (2012a) LRP8 mediates Wnt/beta-catenin signaling and controls osteoblast differentiation. J Bone Miner Res. (In Press)

Zhang, L., Gao, X., Wen, J., Ning, Y., and Chen, Y.G. (2006). Dapper 1 antagonizes Wnt signaling by promoting dishevelled degradation. J Biol Chem 281, 8607-8612.

Zhang, L., Huang, H., Zhou, F., Schimmel, J., Pardo, C.G., Zhang, T., Barakat, T.S., Sheppard, K.A., Mickanin, C., Porter, J.A., et al. (2012b). RNF12 Controls Embryonic Stem Cell Fate and Morphogenesis in Zebrafish Embryos by Targeting Smad7 for Degradation. Mol Cell 46, 650-661.

Zhang, L., Shi, S., Zhang, J., Zhou, F., and ten Dijke, P. Wnt/beta-catenin signaling changes $\mathrm{C} 2 \mathrm{C} 12$ myoblast proliferation and differentiation by inducing Id3 expression. (2012c). Biochem Biophys Res Commun 419, 83-88.

Zhang, L., Zhou, F., van Laar, T., Zhang, J., van Dam, H., and Ten Dijke, P. (2011). Fas-associated factor 1 antagonizes Wnt signaling by promoting beta-catenin degradation. Mol Biol Cell 22, 
1617-1624.

Zhang, L., Zhou, H., Su, Y., Sun, Z., Zhang, H., Zhang, Y., Ning, Y., Chen, Y.G., and Meng, A. (2004). Zebrafish Dpr2 inhibits mesoderm induction by promoting degradation of nodal receptors. Science 306, 114-117.

Zhou, F., Huang, H., and Zhang, L. (2012a). Bisindoylmaleimide I enhances osteogenic differentiation. Protein Cell 3, 311-320.

Zhou, F., Kai, G., Song, B., Ma, T., van Laar, T., Gong, Y., and Zhang,

L. (2012b). The APP intracellular domain (AICD) inhibits Wnt signalling and promotes neurite outgrowth. Biochim Biophys Acta 1823, 1233-1241.

Zhou, F., van Laar, T., Huang, H., and Zhang, L. (2011a). APP and APLP1 are degraded through autophagy in response to proteasome inhibition in neuronal cells. Protein Cell 2, 377-383.

Zhou, F., Zhang, L., van Laar, T., van Dam, H., and Ten Dijke, P. (2011b). GSK3beta inactivation induces apoptosis of leukemia cells by repressing the function of c-Myb. Mol Biol Cell 22, 3533-3540. 